\title{
Fueter's theorem in discrete Clifford analysis
}

\section{Hilde De Ridder ${ }^{* \dagger}$ and Frank Sommen}

\section{Communicated by F. Colombo}

In this paper, we discretize techniques for the construction of axially monogenic functions to the setting of discrete Clifford analysis. Wherefore, we work in the discrete Hermitian Clifford setting, where each basis vector $e_{j}$ is split into a forward and backward basis vector: $e_{j}=e_{j}^{+}+e_{j}^{-}$. We prove a discrete version of Fueter's theorem in odd dimension by showing that for a discrete monogenic function $f\left(\xi_{0}, \xi_{1}\right)$ left-monogenic in two variables $\xi_{0}$ and $\xi_{1}$ and for a left-monogenic $P_{k}(\xi)$, the $m$-dimensional function $\Delta^{k+\frac{m-1}{2}} f\left(\xi_{0}, \xi\right) P_{k}(\xi)$ is in itself left monogenic, that is, a discrete function in the kernel of the discrete Dirac operator. Closely related, we consider a Vekua-type system for the construction of axially monogenic functions. We consider some explicit examples: the discrete axial-exponential functions and the discrete Clifford-Hermite polynomials. Copyright @ 2015 John Wiley \& Sons, Ltd.

Keywords: discrete Clifford analysis; axial monogenicity; Fueter theorem

\section{Introduction}

In 1935, the Swiss mathematician R. Fueter described in his paper [1] a technique to obtain monogenic quaternionic functions, starting from a holomorphic function in the upper half of the complex plane. The technique is the following: consider a holomorphic function $f(z)=u(x, y)+i v(x, y)$ (for $z=x+i y$ ) in some open subset $\Xi$ of the upper half of the complex plane $\mathbb{C}^{+}=\{z=x+i y \in \mathbb{C}: y>0\}$. Then, in the corresponding region, the function

$$
F\left(q_{0}, \underline{q}\right)=\Delta\left(u\left(q_{0},|\underline{q}|\right)+\frac{\underline{q}}{|\underline{q}|} v\left(q_{0},|\underline{q}|\right)\right)
$$

is both left- and right-monogenic with respect to the quaternionic Cauchy-Riemann operator

$$
D=\partial_{q_{0}}+i \partial_{q_{1}}+j \partial_{q_{2}}+k \partial_{q_{3}} .
$$

This classical result was later generalized to $\mathbb{R}_{0, m}$ for $m$ odd by Sce [2], for $m$ even by Qian[3,4], and in [5], Sce's result was generalized by Sommen as follows: if $m$ is an odd positive integer and $P_{k}(\underline{x})$ is a homogeneous monogenic polynomial of degree $k$ in $\mathbb{R}^{m}$, then

$$
\Delta_{x}^{k+\frac{m-1}{2}}\left[\left(u\left(x_{0}, r\right)+\underline{\omega} v\left(x_{0}, r\right)\right) P_{k}(\underline{x})\right]
$$

is also monogenic in $\tilde{\Omega}$.

The goal of this paper is to discretize Sommen's result to the setting of discrete Clifford analysis. Clifford analysis is a natural generalization of complex analysis to higher dimensions. For a detailed description, we refer to [6-8]. For the sake of completion, we recall some of the basic notions. It is constructed by associating to the standard Euclidean space $\mathbb{R}^{m}$ the real Clifford algebra $\mathbb{R}_{0, m}$, generated by the canonical basis $e_{1}, \ldots, e_{m}$. These generators satisfy the multiplication rule $e_{i} e_{j}+e_{j} e_{i}=-2 \delta_{i j}$. For a basis of $\mathbb{R}_{0, m}$, we consider for each set $A=\left\{j_{1}, \ldots, j_{k}\right\} \subseteq\{1, \ldots, m\}$ the element $e_{A}=e_{j_{1}} \ldots e_{j_{k}}$ with $1 \leqslant j_{1}<j_{2}<\ldots<j_{k} \leqslant m$, together with $e_{\emptyset}=1$, the identity element.

The Euclidean space $\mathbb{R}^{m}$ is embedded in $\mathbb{R}_{0, m}$ by identifying the point $\left(x_{1}, \ldots, x_{m}\right) \in \mathbb{R}^{m}$ with the Clifford vector $\underline{x}=\sum_{j=1}^{m} x_{j} e_{j}$. The vector-valued first-order differential operator $\partial_{\underline{x}}=\sum_{j=1}^{m} e_{j} \partial_{x_{j}}$, called the Dirac operator, underlies the notion of monogenicity of a function; a notion which may be considered as the higher-dimensional analogue of holomorphy in the complex plane. More explicitly,

Department of Mathematical Analysis, Faculty of Engineering and Architecture, Ghent University, Building S22, Galglaan 2, 9000 Gent, Belgium 
a function $f$ defined and continuously differentiable in an open region $\Omega \subset \mathbb{R}^{m}$ and taking values in (a subspace of) $\mathbb{R}_{0, m}$ is (left-) monogenic in $\Omega$ if $\partial_{\underline{x}} f=0$ in $\Omega$. Furthermore, because of the fundamental property $\partial_{\underline{x}}^{2}=-\Delta_{\underline{x}}$, monogeneity may, with true right, be considered as a refinement of harmonic analysis.

Discrete Clifford analysis [9-11] has emerged in recent years as both a direct discretization of Euclidean Clifford analysis and a higherdimensional analogue of discrete complex analysis. There are, although, various choices related to the construction of a discrete Dirac operator. We work in the so-called discrete Hermitian setting, where the use of both forward and backward differences in the definition of the discrete Dirac operator $\partial$ translates into a direct factorization of the discrete (star) Laplacian: $\Delta^{*}=\partial^{2}$. As in Euclidean Clifford analysis, the construction of discrete monogenic functions, that is, functions in the kernel of the discrete Dirac operator, is a central topic in discrete Clifford analysis, and various techniques have already been developed or directly discretized: a discrete CauchyKovalevskaya extension theorem, a discrete Fischer decomposition,.... In this paper, we will consider two new techniques, closely related to the CK-extension, resulting in discrete axially monogenic functions.

In section 2, we will start with recalling some choices and basic notions of discrete Clifford analysis. Section 3 contains a first technique to construct axially monogenic functions, by means of a direct discretization of Sommen's result; the main theorem asserts the following.

\section{Theorem 1}

Let $m$ be odd; let $f\left(\xi_{0}, \xi_{1}\right)$ be a (left-)discrete monogenic function in $\xi_{0}, \xi_{1}$ with real Taylor coefficients and let $P_{k}$ be a discrete (left-) monogenic polynomial of degree $k$ in $\xi$, that is

$$
\left(\partial_{0}+\partial_{1}\right) f\left(\xi_{0}, \xi_{1}\right)=0, \quad \partial P_{k}(\xi)=0,
$$

where $\partial=\sum_{j=1}^{m} \partial_{j}$.

Replacing every $\xi_{1}$ by $\xi=\sum_{j=1}^{m} \xi_{j}$ and letting the discrete Laplace operator $\Delta$ act $k+\frac{m-1}{2}$ times on $f\left(\xi_{0}, \xi\right) P_{k}(\xi)$ results again in a discrete (left-) monogenic function, that is

$$
\left(\partial_{0}+\partial\right) \Delta^{k+\frac{m-1}{2}}\left(f\left(\xi_{0}, \xi\right) P_{k}(\xi)\right)=0
$$

Section 4 introduces the concept of discrete axially monogenic functions and the associated Vekua-type system. Application of the previous result leads to the definition of discrete analogues of some special functions, for example, the discrete axially-exponential functions and the discrete Clifford-Hermite polynomials. The former, in turn, lead to discrete versions of the spherical Bessel function, which appears in the theory of the Helmholtz operator, while the latter play a part in solutions to the discrete Heat equation (e.g. [12]), which can be interpreted as an extension of the Dirac equation with an additional time dimension. Clifford-Hermite polynomials are also being used in the definition of continuous wavelet transforms. In the discrete setting, this could be a subject for future investigations.

\section{Preliminaries}

Let $\mathbb{R}^{m}$ be the $m$-dimensional Euclidean space, with orthonormal basis $e_{j}, j=1, \ldots, m$. In the discrete Clifford setting, we consider over this space a uniform lattice $\mathbb{Z}^{m}$ with arbitrary mesh width 1 . A Clifford vector $\underline{x}$ will only be allowed to show integer co-ordinates. We then introduce the traditional one-sided forward and backward difference operators $\Delta_{j}^{ \pm}, j=1, \ldots, m$, respectively acting on a function $f$ as

$$
\Delta_{j}^{+}[f](\underline{x})=f\left(\underline{x}+e_{j}\right)-f(\underline{x}), \quad \Delta_{j}^{-}[f](\underline{x})=f(\underline{x})-f\left(\underline{x}-e_{j}\right) .
$$

With respect to the $\mathbb{Z}^{m}$-neighborhood of $\underline{x}$, the usual definition of the discrete Laplacian then explicitly reads

$$
\Delta^{*}[f](\underline{x})=\sum_{j=1}^{m}\left(f\left(\underline{x}+e_{j}\right)-f\left(\underline{x}-e_{j}\right)\right)-2 m f(\underline{x})=\sum_{j=1}^{m} \Delta_{j}^{+} \Delta_{j}^{-}[f](\underline{x}),
$$

the notation $\Delta^{*}$ referring to this operator being called the 'star Laplacian', because it contains function values at the midpoints of the faces of the cube centered at $\underline{x}$.

The discrete Dirac operator factorizing this star Laplacian is introduced using the so-called Hermitian setting. In this setting, each basis element $e_{j}$ is split into two basis elements $\mathbf{e}_{j}^{+}$and $\mathbf{e}_{j}^{-}$, cf. [9], and we consider the free algebra over $\left\{\mathbf{e}_{j}^{+}, \mathbf{e}_{j}^{-}\right\}$, satisfying the following relations:

$$
\mathbf{e}_{j}^{-} \mathbf{e}_{\ell}^{-}+\mathbf{e}_{\ell}^{-} \mathbf{e}_{j}^{-}=0=\mathbf{e}_{j}^{+} \mathbf{e}_{\ell}^{+}+\mathbf{e}_{\ell}^{+} \mathbf{e}_{j}^{+} \text {and } \mathbf{e}_{j}^{+} \mathbf{e}_{\ell}^{-}+\mathbf{e}_{\ell}^{-} \mathbf{e}_{j}^{+}=\delta_{j \ell,} \quad j, \ell=1, \ldots, m .
$$

Because $e_{j}=\mathbf{e}_{j}^{+}+\mathbf{e}_{j}^{-}$, these conditions imply that $e_{j}^{2}=+1, j=1, \ldots, m$, that is, the Clifford algebra has signature $(m, 0)$. To introduce the discrete Dirac operator, one then combines each difference operator, forward or backward, with the corresponding forward or backward basis vector:

$$
\partial=\sum_{j=1}^{m} \mathbf{e}_{j}^{+} \Delta_{j}^{+}+\mathbf{e}_{j}^{-} \Delta_{j}^{-} .
$$

Observe that indeed $\partial^{2}=\Delta^{*}$. 
Considering the (commuting) difference operators $\Delta_{j}^{ \pm}$as lowering operators, we introduce (see [10]) the corresponding raising operators $X_{j}^{ \pm}(j=1, \ldots, m)$ satisfying the 'skew' Weyl relations:

$$
\Delta_{j}^{+} X_{j}^{+}-X_{j}^{-} \Delta_{j}^{-}=1=\Delta_{j}^{-} X_{j}^{-}-X_{j}^{+} \Delta_{j}^{+}
$$

which replace the classical Weyl relations holding in the continuous case for the partial derivatives and the vector variable. The operators $X_{j}^{ \pm}$mutually commute and they commute also with $\Delta_{k}^{ \pm}$for $j \neq k$.

The traditional vector variable corresponding to the Dirac operator then is replaced by the operator

$$
\xi=\sum_{j=1}^{m} \mathbf{e}_{j}^{+} X_{j}^{-}+\mathbf{e}_{j}^{-} X_{j}^{+}
$$

of which the components $X_{j}^{ \pm}$are no longer independent, but interconnected by (1). Next, the discrete Euler operator $\mathbb{E}$ ( see also [10]) is defined by imposing the intertwining relation $\partial \xi+\xi \partial=2 \mathbb{E}+m$, which holds for the Dirac operator and the vector variable in the continuous Clifford case. This discrete Euler operator $\mathbb{E}$ is then easily seen to satisfy also the other usual intertwining relations with the Dirac operator and the vector variable, respectively, that is, $\partial \mathbb{E}=\mathbb{E} \partial+\partial$ and $\mathbb{E} \xi=\xi \mathbb{E}+\xi$. The notion of homogeneity of a discrete polynomial is then defined as follows.

\section{Definition 1}

A discrete polynomial $P_{k}$ is called discrete homogeneous of degree $k$ if and only if it is an eigenfunction with eigenvalue $k$ of the discrete Euler operator: $\mathbb{E} P_{k}=k P_{k}$.

Introduction of the co-ordinate variable $\xi_{j}=X_{j}^{+} \mathbf{e}_{j}^{-}+X_{j}^{-} \mathbf{e}_{j}^{+}, j=1, \ldots, m$ and of the co-ordinate difference operator $\partial_{j}=\mathbf{e}_{j}^{+} \Delta_{j}^{+}+$ $\mathbf{e}_{j}^{-} \Delta_{j}^{-}, j=1, \ldots, m$, enables us to decompose the discrete Dirac operator and the vector variable respectively as $\partial=\sum_{j=1}^{m} \partial_{j}$ and $\xi=\sum_{j=1}^{m} \xi_{j}$. On account of the skew Weyl relations (1), it is easily seen that $\xi_{j}$ and $\partial_{j}$ satisfy the graded (anti-)commuting relations

$$
\partial_{j} \xi_{j}-\xi_{j} \partial_{j}=1, j=1, \ldots, m, \quad \text { and } \partial_{\ell} \xi_{j}+\xi_{j} \partial_{\ell}=0, \ell \neq j, j, \ell=1, \ldots, m .
$$

However, the $\xi_{j}$ are mutually anti-commuting $\left(\xi_{j} \xi_{k}=-\xi_{k} \xi_{j}\right)$ as are the difference operators $\left(\partial_{j} \partial_{k}=-\partial_{k} \partial_{j}\right.$ for $\left.j \neq k\right)$.

Moreover, using the intertwining relation $\mathbb{E} \xi=\xi(\mathbb{E}+1)$, it directly follows that $\mathbb{E} \xi_{j}=\xi_{j}(\mathbb{E}+1)$, whence $\xi_{j}^{k}[1]$, that is, natural powers of the operator $\xi_{j}$ acting on the ground state 1, are the basic discrete homogeneous polynomials of degree $k$ in the variable $x_{j}$, similar to the basic homogeneous powers $x_{j}^{k}$ in the continuous setting. A closed form for these polynomials was obtained in [10]; they are given by

$$
\begin{gathered}
\xi_{j}^{2 n+1}[1]\left(x_{j}\right)=x_{j} \prod_{i=1}^{n}\left(x_{j}^{2}-i^{2}\right)\left(\mathbf{e}_{j}^{+}+\mathbf{e}_{j}^{-}\right), \\
\xi_{j}^{2 n}[1]\left(x_{j}\right)=\left(x_{j}^{2}+n x_{j}\left(\mathbf{e}_{j}^{+} \wedge \mathbf{e}_{j}^{-}\right)\right) \prod_{i=1}^{n-1}\left(x_{j}^{2}-i^{2}\right),
\end{gathered}
$$

for each $n=1,2, \ldots$ and $j=1, \ldots, m$. Here, $\mathbf{e}_{j}^{+} \wedge \mathbf{e}_{j}^{-}$denotes $\mathbf{e}_{j}^{+} \mathbf{e}_{j}^{-}-\mathbf{e}_{j}^{-} \mathbf{e}_{j}^{+}$. Note that a 'discrete homogeneous polynomial' is not homogeneous in the classical sense, because the property $\xi_{j}^{k}[1]\left(t x_{j}\right)=t^{k} \xi_{j}^{k}[1]\left(x_{j}\right)$ does not hold.

A function defined on $\mathbb{Z}^{m}$ is then called (left-) discrete monogenic in a domain $\Omega \subset \mathbb{Z}^{m}$ if it satisfies in $\Omega$ the equation $\partial f=0$, or, in other words, if it is a null solution for the left action of the discrete Dirac operator. Discrete homogeneous polynomial null solutions of $\partial$ are called discrete spherical monogenics, and the space of discrete spherical monogenics of degree $k$ is denoted $\mathcal{M}_{k}$. We adopted the name 'discrete spherical monogenic' to indicate that these functions are the discrete analogues of the spherical monogenics, a notion which is standard in Clifford analysis. This does not mean that they can be defined on a discrete version of the sphere.

\section{Generalized Fueter's theorem}

Before discussing the discrete Fueter theorem (Theorem 1), we will prove some auxiliary lemmas.

Lemma 1

The repeated action of $\partial$ on terms of the form $\xi^{s} P_{k}(\xi)$ is given by

$$
\begin{array}{rlrl}
\partial^{2 \ell} \xi^{2 i} P_{k}(\xi) & =\frac{(2 i) ! !}{(2 i-2 \ell) ! !} \frac{(2 i+2 k+m-2) ! !}{(2 i+2 k+m-2 \ell-2) ! !} \xi^{2 i-2 \ell} P_{k}(\xi), & \forall \ell \leqslant i, & \\
\partial^{2 \ell+1} \xi^{2 i} P_{k}(\xi) & =\frac{(2 i) ! !}{(2 i-2 \ell-2) ! !} \frac{(2 i+2 k+m-2) ! !}{(2 i+2 k+m-2 \ell-2) ! !} \xi^{2 i-2 \ell-1} P_{k}(\xi), \quad \forall \ell \leqslant i-1, \\
\partial^{2 \ell} \xi^{2 i+1} P_{k}(\xi) & =\frac{(2 i) ! !}{(2 i-2 \ell) ! !} \frac{(2 i+2 k+m) ! !}{(2 i+2 k+m-2 \ell) ! !} \xi^{2 i-2 \ell+1} P_{k}(\xi), & \forall \ell \leqslant i, & \\
\partial^{2 \ell+1} \xi^{2 i+1} P_{k}(\xi) & =\frac{(2 i) ! !}{(2 i-2 \ell) ! !} \frac{(2 i+2 k+m) ! !}{(2 i+2 k+m-2 \ell-2) ! !} \xi^{2 i-2 \ell} P_{k}(\xi), & \forall \ell \leqslant i .
\end{array}
$$


Proof

We take into account the basic rules (with $P_{k}$ a discrete spherical monogenic of degree $k$ in $\xi$ ):

and apply a recursion argument.

$$
\begin{gathered}
\partial \xi^{2 i} P_{k}(\xi)=2 i \xi^{2 i-1} P_{k} \\
\partial \xi^{2 i+1} P_{k}(\xi)=(2 i+2 k+m) \xi^{2 i} P_{k}
\end{gathered}
$$

Now, let $m=2 n+1$ be odd. Consider a discrete monogenic function $f\left(\xi_{0}, \xi_{1}\right)$ in two variables with real Taylor coëfficiënts, this function can be written as (for example [13])

$$
f=\sum_{\ell=0}^{\infty} \underbrace{z_{1} \hat{z}_{1} z_{1} \ldots}_{\ell} c_{\ell}, \quad c_{\ell} \in \mathbb{R} .
$$

Here, $z_{1}=\xi_{1}-\xi_{0}$ and $\hat{z}_{1}=\xi_{1}+\xi_{0}$. Denote $\xi=\sum_{j=1}^{m} \xi_{j}$ and let $P_{k}(\xi) \in \mathcal{M}_{k}$ be a discrete spherical monogenic of degree $k$. Then, we will show that $\Delta^{k+\frac{m-1}{2}}\left(f\left(\xi_{0}, \xi\right) P_{k}(\xi)\right)$ is a discrete monogenic function in $m+1$ variables $\xi_{0}, \ldots, \xi_{m}$, that is

$$
\left(\partial_{0}+\partial\right) \Delta^{k+\frac{m-1}{2}}\left(f\left(\xi_{0}, \xi\right) P_{k}(\xi)\right)=\left(\partial_{0}+\partial\right)^{2 k+m}\left(f\left(\xi_{0}, \xi\right) P_{k}(\xi)\right)=0
$$

Because a discrete monogenic function in two variables with real Taylor coefficients is a linear combination of terms of the form $\left(z_{1} \hat{z}_{1}\right)^{\ell}$ and $\left(z_{1} \hat{z}_{1}\right)^{\ell} z_{1}$, we can consider both options separately.

First, take $f=\left(z_{2} \hat{z}_{2}\right)^{\ell}$. We will show that

$$
\left(\partial_{0}+\partial\right)^{2 k+2 n+1}\left[\left(\left(\xi-\xi_{0}\right)\left(\xi+\xi_{0}\right)\right)^{\ell} P_{k}(\xi)\right]=0 .
$$

Therefore, we use that

$$
\left(\partial_{0}+\partial\right)^{2 k+2 n+1}=\sum_{i=0}^{k+n}\left(\begin{array}{c}
k+n \\
i
\end{array}\right)\left(\partial_{0}^{2 i+1} \partial^{2 k+2 n-2 i}+\partial_{0}^{2 i} \partial^{2 k+2 n-2 i+1}\right)
$$

and

$$
\left(\left(\xi-\xi_{0}\right)\left(\xi+\xi_{0}\right)\right)^{\ell}=\sum_{j=0}^{\ell}(-1)^{\ell+j}\left(\begin{array}{c}
2 \ell \\
2 j
\end{array}\right) \xi_{0}^{2 \ell-2 j} \xi^{2 j}+\sum_{j=0}^{\ell-1}(-1)^{\ell+j}\left(\begin{array}{c}
2 \ell \\
2 j+1
\end{array}\right) \xi_{0}^{2 \ell-2 j-1} \xi^{2 j+1}
$$

(for example [14]). Combining both expressions then shows that

$$
\begin{aligned}
& \left(\partial_{0}+\partial\right)^{2 k+2 n+1}\left[\left(\left(\xi-\xi_{0}\right)\left(\xi+\xi_{0}\right)\right)^{\ell} P_{k}(\xi)\right] \\
& =\sum_{i=0}^{k+n} \sum_{j=0}^{\ell}(-1)^{\ell+j}\left(\begin{array}{c}
k+n \\
i
\end{array}\right)\left(\begin{array}{c}
2 \ell \\
2 j
\end{array}\right) \partial_{0}^{2 i+1} \partial^{2 k+2 n-2 i}\left(\xi_{0}^{2 \ell-2 j} \xi^{2 j} P_{k}(\xi)\right) \\
& \quad+\sum_{i=0}^{k+n} \sum_{j=0}^{\ell-1}(-1)^{\ell+j}\left(\begin{array}{c}
k+n \\
i
\end{array}\right)\left(\begin{array}{c}
2 \ell \\
2 j+1
\end{array}\right) \partial_{0}^{2 i+1} \partial^{2 k+2 n-2 i}\left(\xi_{0}^{2 \ell-2 j-1} \xi^{2 j+1} P_{k}(\xi)\right) \\
& +\sum_{i=0}^{k+n} \sum_{j=0}^{\ell}(-1)^{\ell+j}\left(\begin{array}{c}
k+n \\
i
\end{array}\right)\left(\begin{array}{c}
2 \ell \\
2 j
\end{array}\right) \partial_{0}^{2 i} \partial^{2 k+2 n-2 i+1}\left(\xi_{0}^{2 \ell-2 j} \xi^{2 j} P_{k}(\xi)\right) \\
& \quad+\sum_{i=0}^{k+n} \sum_{j=0}^{\ell-1}(-1)^{\ell+j}\left(\begin{array}{c}
k+n \\
i
\end{array}\right)\left(\begin{array}{c}
2 \ell \\
2 j+1
\end{array}\right) \partial_{0}^{2 i} \partial^{2 k+2 n-2 i+1}\left(\xi_{0}^{2 \ell-2 j-1} \xi^{2 j+1} P_{k}(\xi)\right)
\end{aligned}
$$

Applying Lemma 1 and $\partial_{0}^{s} \xi_{0}^{k}=\frac{k !}{(k-s) !} \xi_{0}^{k-s}$, we find that

$$
\begin{aligned}
& \left(\partial_{0}+\partial\right)^{2 k+2 n+1}\left[\left(\left(\xi-\xi_{0}\right)\left(\xi+\xi_{0}\right)\right)^{\ell} P_{k}(\xi)\right] \\
& =\sum_{i=0}^{k+n} \sum_{j=0}^{\ell}(-1)^{\ell+j}\left(\begin{array}{c}
k+n \\
i
\end{array}\right)\left(\begin{array}{c}
2 \ell \\
2 j
\end{array}\right) \frac{(2 \ell-2 j) !}{(2 \ell-2 j-2 i-1) !} \frac{(2 j) ! !}{(2 j-2 k-2 n+2 i) ! !} \\
& \quad \frac{(2 j+2 k+m-2) ! !}{(2 j+2 k+m-(2 k+2 n-2 i)-2) ! !} \xi_{0}^{2 \ell-2 j-2 i-1} \xi^{2 j-2 k-2 n+2 i} P_{k} \\
& +\sum_{i=0}^{k+n} \sum_{j=0}^{\ell-1}\left(\begin{array}{c}
(-1)^{\ell+j}\left(\begin{array}{c}
k+n \\
i
\end{array}\right)\left(\begin{array}{c}
2 \ell \\
2 j+1
\end{array}\right) \frac{(2 \ell-2 j-1) !}{(2 \ell-2 j-2 i-2) !} \frac{(2 j) ! !}{(2 j-2 k-2 n+2 i) ! !} \\
\frac{(2 j+2 k+m) ! !}{(2 j+2 k+m-(2 k-2 n-2 i)) ! !} \xi_{0}^{2 \ell-2 j-2 i-2} \xi^{2 j-2 k-2 n+2 i+1} P_{k}
\end{array}\right.
\end{aligned}
$$




$$
\begin{aligned}
& +\sum_{i=0}^{k+n} \sum_{j=0}^{\ell}(-1)^{\ell+j}\left(\begin{array}{c}
k+n \\
i
\end{array}\right)\left(\begin{array}{c}
2 \ell \\
2 j
\end{array}\right) \frac{(2 \ell-2 j) !}{(2 \ell-2 j-2 i) !} \frac{(2 j) ! !}{(2 j-2 k-2 n+2 i-2) ! !} \\
& \frac{(2 j+2 k+m-2) ! !}{(2 j+2 k+m-(2 k+2 n-2 i)-2) ! !} \xi_{0}^{2 \ell-2 j-2 i} \xi^{2 j-2 k-2 n+2 i-1} P_{k} \\
& +\sum_{i=0}^{k+n} \sum_{j=0}^{\ell-1}(-1)^{\ell+j+1}\left(\begin{array}{c}
k+n \\
i
\end{array}\right)\left(\begin{array}{c}
2 \ell \\
2 j+1
\end{array}\right) \frac{(2 \ell-2 j-1) !}{(2 \ell-2 j-2 i-1) !} \frac{(2 j) ! !}{(2 j-2 k-2 n+2 i) ! !} \\
& \frac{(2 j+2 k+m) ! !}{(2 j+2 k+m-(2 k+2 n-2 i)-2) ! !} \xi_{0}^{2 \ell-2 j-2 i-1} \xi^{2 j-2 k-2 n+2 i} P_{k} .
\end{aligned}
$$

The extra minus sign in line (7) arises due to the anti-commutativity of $\xi_{0}$ and $\xi$. We will now look directly at the level of the coefficients. Note that in (4) and (7), the powers of $\xi_{0}$ are odd and the powers of $\xi$ are even while in (5) and (6), the powers of $\xi_{0}$ are even and the powers of $\xi$ are odd.

We now consider (4) and (7): note that the only non-vanishing values for $j$ are $j \in\{k+n-i, \ldots, \ell-i-1\}$ because, otherwise, the operators $\partial_{0}$ and $\partial$ act on constants, resulting in zero. Note that a realistic assumption on the value of $\ell$ is that $\ell>\left\lfloor n+\frac{k}{2}\right\rfloor$ because for lower values of $\ell$, the action of $\left(\partial_{0}+\partial\right)^{2 k+2 n+1}$ on the homogeneous polynomial $\left(z_{2} \hat{z}_{2}\right)^{\ell} P_{k}$ of degree $2 \ell+k$ will automatically give zero.

The (odd) powers of $\xi_{0}$ range from 1 to $2 \ell-2 k-2 n-1$ while the (even) powers of $\xi$ range from 0 to $2 \ell-2 k-2 n-2$. We now consider the coefficient of $\xi_{0}^{2 s+1} \xi^{2 \ell-2 k-2 n+2 s-2} P_{k}$, with $s=0, \ldots, \ell-k-n-1$. Therefore, we choose $j=\ell-i-s-1$ in both lines (4) and (7):

$$
\begin{aligned}
\sum_{i=0}^{k+n} & \left(\begin{array}{c}
k+n \\
i
\end{array}\right)(2 \ell) !(-1)^{i-s-1} \\
& {\left[\frac{1}{(2 \ell-2 i-2 s-2) !(2 s+1) !} \frac{(2 \ell-2 i-2 s-2) ! !}{(2 \ell-2 s-2 k-2 n-2) ! !} \frac{(2 \ell-2 i-2 s+2 k+2 n-3) ! !}{(2 \ell-2 s-3) ! !}\right.} \\
& \left.-\frac{1}{(2 \ell-2 i-2 s-1) !(2 s+1) !} \frac{(2 \ell-2 i-2 s-2) ! !}{(2 \ell-2 s-2 k-2 n-2) ! !} \frac{(2 \ell-2 i-2 s+2 k+2 n-1) ! !}{(2 \ell-2 s-3) ! !}\right] \\
= & (-1)^{s+1} \frac{(2 \ell) !}{(2 s+1) !(2 \ell-2 s-3) ! !} \sum_{i=0}^{k+n}\left(\begin{array}{c}
k+n \\
i
\end{array}\right)(-1)^{i} \frac{(2 \ell-2 i-2 s-2) ! !}{(2 \ell-2 s-2 k-2 n-2) ! !} \\
& \frac{(2 \ell-2 i-2 s+2 k+2 n-3) ! !}{(2 \ell-2 i-2 s-2) !}\left[\begin{array}{c}
\left.1-\frac{(2 \ell-2 i-2 s+2 k+2 n-1)}{(2 \ell-2 i-2 s-1)}\right] \\
=
\end{array}\right. \\
& \frac{2(-1)^{s} \frac{2(k+n)(2 \ell) !}{(2 s+1) !} \frac{2^{\ell-s-1}(\ell-s-1) !}{(2 \ell-2 s-2) !} \sum_{i=0}^{k+n}\left(\begin{array}{c}
k+n \\
2^{\ell-s-k-n-1}(\ell-s-k-n-1) !
\end{array}\right)(-1)^{i}}{2^{\ell-i-s+k+n-1}(\ell-i-s+k+n-1) !(2 \ell-2 i-2 s-1) !}(\ell-i-s-1) ! \\
= & (-1)^{s} \frac{2(k+n)(2 \ell) !}{(2 s+1) !} \frac{(\ell-s-1) !}{(2 \ell-2 s-2) !} \sum_{i=0}^{k+n}\left(\begin{array}{c}
k+n \\
i
\end{array}\right)(-1)^{i} \\
& \frac{(\ell-i-s-1) !}{(\ell-s-k-n-1) !} \frac{(2 \ell-2 i-2 s+2 k+2 n-2) !}{(\ell-i-s+k+n-1) !(2 \ell-2 i-2 s-1) !} .
\end{aligned}
$$

Here, we used that $(2 j) ! !=2^{j} j !$ and $(2 j-1) ! !=\frac{(2 j) !}{2^{j} j !}$. Now, one can check by means of symbolic computation software, for example, Maple (Maplesoft, Waterloo, Ontario, Canada), that this is zero.

Looking at the line (5), we may note that the only non-vanishing values of $j$ are those in the rang $\{k-n+i, \ldots, \ell-i-1\}$, while the $j$-values in line (6) must be taken in $\{k-n+i+1, \ldots, \ell-i\}$. We may further conclude that the powers of $\xi_{0}$ are even and range between 0 and $2 \ell-2 k-2 n-2$ while the powers of $\xi$ are odd and range between 1 and $2 \ell-2 k-2 n-1$ :

$$
\begin{gathered}
\sum_{i=0}^{k+n} \sum_{j=k+n-i}^{\ell-i-1}(-1)^{\ell+j}\left(\begin{array}{c}
k+n \\
i
\end{array}\right)\left(\begin{array}{c}
2 \ell \\
2 j+1
\end{array}\right) \frac{(2 \ell-2 j-1) !}{(2 \ell-2 j-2 i-2) !} \frac{(2 j) ! !}{(2 j-2 k-2 n+2 i) ! !} \\
\frac{(2 j+2 k+2 n+1) ! !}{(2 j+2 i+1) ! !} \xi_{0}^{2 \ell-2 j-2 i-2} \xi^{2 j-2 k-2 n+2 i+1} P_{k} \\
+\sum_{i=0}^{k+n} \sum_{j=k+n-i+1}^{\ell-i}(-1)^{\ell+j}\left(\begin{array}{c}
k+n \\
i
\end{array}\right)\left(\begin{array}{c}
2 \ell \\
2 j
\end{array}\right) \frac{(2 \ell-2 j) !}{(2 \ell-2 j-2 i) !} \frac{(2 j) ! !}{(2 j-2 k-2 n+2 i-2) ! !} \\
\frac{(2 j+2 k+2 n-1) ! !}{(2 j+2 i-1) ! !} \xi_{0}^{2 \ell-2 j-2 i} \xi^{2 j-2 k-2 n+2 i-1} P_{k} .
\end{gathered}
$$


If we now only consider the coefficient of $\xi^{2 s} \xi^{2 \ell-2 k-2 n-2 s-1} P_{k}$ with $s \in\{0, \ldots, l-k-n-1\}$ we must choose $j=\ell-i=s-1$ in the first term and $j=\ell-i-s$ in the second term (for each $i=0, \ldots, k+n$ ):

$$
\begin{aligned}
& \sum_{i=0}^{k+n}(-1)^{i-s-1}\left(\begin{array}{c}
k+n \\
i
\end{array}\right)\left(\begin{array}{c}
2 \ell \\
2 \ell-2 i-2 s-1
\end{array}\right) \frac{(2 i+2 s+1) !}{(2 s) !} \frac{(2 \ell-2 i-2 s-2) ! !}{(2 \ell-2 s-2 k-2 n-2) ! !} \\
& \frac{(2 \ell-2 i-2 s+2 k+2 n-1) ! !}{(2 \ell-2 s-1) ! !} \\
& +\sum_{i=0}^{k+n}(-1)^{i-s}\left(\begin{array}{c}
k+n \\
i
\end{array}\right)\left(\begin{array}{c}
2 \ell \\
2 \ell-2 i-2 s
\end{array}\right) \frac{(2 i+2 s) !}{(2 s) !} \frac{(2 \ell-2 i-2 s) ! !}{(2 \ell-2 s-2 k-2 n-2) ! !} \\
& \quad \frac{(2 \ell-2 i-2 s+2 k+2 n-1) ! !}{(2 \ell-2 s-1) ! !} \\
& =\sum_{i=0}^{k+n}(-1)^{i-s-1}\left(\begin{array}{c}
k+n \\
i
\end{array}\right) \frac{(2 \ell) !}{(2 \ell-2 i-2 s-1) !(2 s) !} \frac{(2 \ell-2 i-2 s+2 k+2 n-1) ! !}{(2 \ell-2 s-1) ! !} \\
& \frac{(2 \ell-2 i-2 s-2) ! !}{(2 \ell-2 s-2 k-2 n-2) ! !}\left[\begin{array}{c}
\left.1-\frac{(2 \ell-2 i-2 s)}{(2 \ell-2 i-2 s)}\right] \\
(2 \ell-2 i-2 k
\end{array}\right.
\end{aligned}
$$

The second option for the monogenic function $f, f=\left(z_{2} \hat{z}_{2}\right)^{\ell} z_{2}$, is considered completely analogous.

\section{Discrete axial monogenic functions}

Fueter's theorem results in functions that are axially symmetric around the $\xi_{0}$-axis. A second method of obtaining such axially monogenic functions is to consider monogenic functions of the form

$$
\left(A\left(\xi_{0}, \xi^{2}\right)+\xi B\left(\xi_{0}, \xi^{2}\right)\right) P_{k}(\xi)
$$

where

$$
A=\sum_{\ell, s=0}^{\infty} \xi_{0}^{s} \xi^{2 \ell} a_{s, \ell}, \quad B=\sum_{\ell, s=0}^{\infty} \xi_{0}^{s} \xi^{2 \ell} b_{s, \ell}
$$

are polynomial operators with real coefficients $a_{s, \ell}, b_{s, \ell}$ acting on a discrete homogeneous monogenic $P_{k}(\xi)$ of degree $k$ in $\mathbb{Z}^{m}$, that is

$$
\partial P_{k}(\xi)=\sum_{j=1}^{m} \partial_{j} P_{k}(\xi)=0, \quad \quad \mathbb{E} P_{k}(\xi)=\sum_{j=1}^{m} \xi_{j} \partial_{j} P_{k}(\xi)=k P_{k}(\xi)
$$

These functions are discrete analogues of the classic axial monogenic functions in Euclidean Clifford analysis, in which case they generate all monogenic functions defined in axially symmetric domains. However, the functions $A\left(\xi_{0}, \xi^{2}\right)$ and $B\left(\xi_{0}, \xi^{2}\right)$ can no longer be considered scalar-valued, because the operator $\xi^{2}$ contains both a scalar and a bivector-part.

Remark 1

One has that

$$
\partial\left(\xi^{2 \ell+1} P_{k}\right)=(2 \ell+2 k+m) \xi^{2 \ell} P_{k \prime} \quad \partial\left(\xi^{2 \ell+1}[1]\right)=(2 \ell+m) \xi^{2 \ell}[1] .
$$

We may thus consider the term $(2 \ell+m) \xi^{2 \ell}$ as the formal derivative of the operator $\xi^{2 \ell+1}$. We will denote this as $\frac{d}{d \xi} \xi^{2 \ell+1}$; it is again an operator, satisfying

$$
\frac{d}{d \xi} \xi^{2 \ell+1}[1]=\partial \xi^{2 \ell+1}[1]
$$

This means that when acting on the trivial discrete monogenic 1, the formal derivative is nothing else than the normal derivative. However, when acting on a more general discrete monogenic $P_{k}$, we see that

$$
\partial\left(\xi^{2 \ell+1} P_{k}\right)=\frac{d}{d \xi}\left(\xi^{2 \ell+1}\right) P_{k}+2 k \xi^{2 \ell} P_{k} .
$$

Because $\partial \xi^{2 \ell} P_{k}=2 \ell \xi^{2 \ell-1} P_{k}$, for all discrete monogenic polynomials $P_{k}$, we define the formal derivative of $\xi^{2 \ell}$ as

$$
\frac{d}{d \xi} \xi^{2 \ell}=2 \ell \xi^{2 \ell-1}
$$

In conclusion, the formal derivative of a polynomial operator $A=\sum_{k=0}^{\infty} \xi^{k} a_{k}$ is 


$$
\frac{d}{d \xi}(A)=\sum_{k=0}^{\infty} 2 k \xi^{2 k-1} a_{2 k}+\sum_{k=0}^{\infty}(2 k+m) \xi^{2 k} a_{2 k+1}
$$

Note that $\frac{d}{d \xi}(A)[1]=\partial(A[1])$.

Similarly, we denote $\frac{d}{d \xi_{0}} \xi_{0}^{k}=k \xi_{0}^{k-1}$; again we may remark that $\frac{d}{d \xi_{0}} \xi_{0}^{k}[1]=\partial \xi_{0}^{k}[1]$.

We thus find that

$$
\partial\left(A\left(\xi_{0}, \xi^{2}\right) P_{k}\right)=\sum_{s, \ell=0}^{\infty}(-1)^{s}(2 \ell) a_{s, \ell} \xi_{0}^{s} \xi^{2 \ell-1} P_{k}=\frac{d}{d \xi}(A) P_{k}
$$

Furthermore, we use $\partial \xi^{2 \ell+1} P_{k}=(2 \ell+2 k+m) \xi^{2 \ell} P_{k}$ to show that

$$
\partial\left(\xi B\left(\xi_{0}, \xi^{2}\right) P_{k}(\xi)\right)=\sum_{s, \ell=0}^{\infty}(2 \ell+2 k+m) \xi_{0}^{s} \xi^{2 \ell} P_{k}=(2 k+m) B P_{k}+\xi \frac{d}{d \xi}(B) P_{k}
$$

The given function $(A+\xi B) P_{k}$ is thus monogenic if

$$
0=\left(\partial_{0}+\partial\right)(A+\xi B) P_{k}=\left[\frac{d}{d \xi_{0}}(A)-\xi \frac{d}{d \xi_{0}}(B)+\frac{d}{d \xi}(A)+(2 k+m) B+\xi \frac{d}{d \xi}(B)\right] P_{k},
$$

or equivalently if they satisfy the Vekua-type system

$$
\left\{\begin{array}{l}
\frac{d}{d \xi_{0}}(A)+\xi \frac{d}{d \xi}(B)+(2 k+m) B=0 \\
\frac{d}{d \xi}(A)-\xi \frac{d}{d \xi_{0}}(B)=0
\end{array}\right.
$$

Remark 2

Note that Fueter's theorem generates axial monogenic functions. Indeed, replacing $\xi_{1}$ by $\xi$ in the Taylor series of $f\left(\xi_{0}, \xi_{1}\right)$, and letting $\Delta$ act $\frac{m}{2}$ times on this Taylor series will result in a monogenic function of the form

$$
A\left(\xi_{0}, \xi^{2}\right)+\xi B\left(\xi_{0}, \xi^{2}\right)
$$

Remark 3

The Vekua-system can equivalently be expressed as

$$
\left\{\begin{array}{l}
\left(\partial_{0} A+\xi \partial B+(2 k+m) B\right)[1]=0, \\
\left(\partial A-\xi \partial_{0} B\right)[1]=0 .
\end{array}\right.
$$

As corollary of (2)-(3), it holds that

$$
\forall x_{i} \in \mathbb{Z}, \forall k \geqslant 2\left|x_{i}\right|+1: \xi_{i}^{k}\left(x_{i}\right)=0,
$$

implying that the convergence of series of the form $\sum_{s} a_{s} \xi^{s}$ is never an issue, because for each point $\left(x_{1}, \ldots, x_{m}\right) \in \mathbb{Z}$, there exists a natural number $N$ such that $\xi^{s}[1]\left(x_{1}, \ldots, x_{m}\right)=0$, for all $s \geqslant N$. In each point of the grid, the series will reduce to a finite sum.

Example 1

We consider the example where $A\left(\xi_{0}, \xi^{2}\right)=e^{\xi_{0}} a\left(\xi^{2}\right)$ and $B\left(\xi_{0}, \xi^{2}\right)=e^{\xi_{0}} b\left(\xi^{2}\right)$, where $a(x)$ and $b(x)$ are real-valued functions, that is

$$
a\left(\xi^{2}\right)=\sum_{s=0}^{\infty} \xi^{2 s} a_{s,} \quad b\left(\xi^{2}\right)=\sum_{s=0}^{\infty} \xi^{2 s} b_{s,}
$$

with $a_{s}, b_{s} \in \mathbb{R}$. It is obvious that $\frac{d}{d \xi_{0}}\left(A\left(\xi_{0}, \xi^{2}\right)\right)=A\left(\xi_{0}, \xi^{2}\right)$ and $\frac{d}{d \xi_{0}}\left(B\left(\xi_{0}, \xi^{2}\right)\right)=B\left(\xi_{0}, \xi^{2}\right)$. Furthermore

$$
\frac{d}{d \xi}\left(A\left(\xi_{0}, \xi^{2}\right)\right)=e^{-\xi_{0}} \frac{d}{d \xi}\left(a\left(\xi^{2}\right)\right), \quad \frac{d}{d \xi}\left(B\left(\xi_{0}, \xi^{2}\right)\right)=e^{-\xi_{0}} \frac{d}{d \xi}(b) .
$$

We, thus, obtain the following system

$$
\left\{\begin{array}{l}
e^{\xi_{0}} a+\xi e^{-\xi_{0}} \frac{d}{d \xi}(b)+(2 k+m) e^{\xi_{0}} b=0 \\
e^{-\xi_{0}} \frac{d}{d \xi}(a)-\xi e^{\xi_{0}} b=0
\end{array}\right.
$$


which can be rewritten as

$$
\left\{\begin{array} { l } 
{ e ^ { \xi _ { 0 } } a + e ^ { \xi _ { 0 } } \xi \frac { d } { d \xi } ( b ) + ( 2 k + m ) e ^ { \xi _ { 0 } } b = 0 } \\
{ e ^ { - \xi _ { 0 } } \frac { d } { d \xi } ( a ) - e ^ { - \xi _ { 0 } } \xi b = 0 }
\end{array} \Leftrightarrow \left\{\begin{array}{l}
a+\xi \frac{d}{d \xi}(b)+(2 k+m) b=0 \\
\frac{d}{d \xi}(a)-\xi b=0 .
\end{array}\right.\right.
$$

Looking at the coefficients, we see that the second equation implies that

$$
\sum_{s=1}^{\infty}(2 s) \xi^{2 s-1} a_{s}=\sum_{p=0}^{\infty} \xi^{2 p+1} b_{p} \Leftrightarrow \sum_{p=0}^{\infty}(2 p+2) \xi^{2 p+1} a_{p+1}=\sum_{p=0}^{\infty} \xi^{2 p+1} b_{p}
$$

or thus $(2 p+2) a_{p+1}=b_{p}$, for $p=0,1, \ldots$. We now consider the first equation on the level of the coefficients:

$$
\sum_{s=0}^{\infty} \xi^{2 s} a_{s}+\sum_{s=0}^{\infty}(2 s) \xi^{2 s} b_{s}+(2 k+m) \sum_{s=0}^{\infty} \xi^{2 s} b_{s}=0
$$

or thus for all $s=0,1, \ldots$ :

$$
a_{s}+(2 s) b_{s}+(2 k+m) b_{s}=0 \Leftrightarrow a_{s}+(2 s) b_{s}+(2 k+m) b_{s}=0 .
$$

For $s=0$, this shows that $a_{0}+(2 k+m) b_{0}=0$. For $s \geqslant 1$, we obtain that

$$
\begin{aligned}
\frac{b_{s-1}}{2 s}+(2 s) b_{s}+(2 k+m) b_{s}=0 & \Leftrightarrow \quad b_{s-1}=-(2 s)(2 k+2 s+m) b_{s} \\
& \Leftrightarrow \quad b_{s}=\frac{-b_{s-1}}{(2 s)(2 k+2 s+m)} .
\end{aligned}
$$

Set $b_{0}=C$, then one finds recursively that

$$
b_{s}=\frac{(-1)^{s}(2 k+m) ! !}{(2 s) ! !(2 k+m+2 s) ! !} C \text {. }
$$

From here, it follows easily that

$$
\begin{aligned}
& a_{0}=-(2 k+m) b_{0}=-(2 k+m) C \\
& a_{s}=\frac{1}{2 s} b_{s-1}=\frac{(-1)^{s-1}(2 k+m) ! !}{(2 s) ! !(2 k+m+2 s-2) ! !} C .
\end{aligned}
$$

Now consider $m$ to be even. Then

$$
a_{s}=\frac{(-1)^{s-1}}{2^{s} s !} \frac{2^{k+\frac{m}{2}}\left(k+\frac{m}{2}\right) !}{2^{k+\frac{m}{2}+s-1}\left(k+\frac{m}{2}+s-1\right) !} C=\frac{(-1)^{s-1}}{2^{2 s-1} s !} \frac{\Gamma\left(k+\frac{m}{2}+1\right)}{\Gamma\left(k+\frac{m}{2}+s\right)} C
$$

and

$$
b_{s}=\frac{(-1)^{s}}{2^{s} s !} \frac{2^{k+\frac{m}{2}}\left(k+\frac{m}{2}\right) !}{2^{k+\frac{m}{2}+s}\left(k+\frac{m}{2}+s\right) !} C=\frac{(-1)^{s}}{2^{2 s} s !} \frac{\Gamma\left(k+\frac{m}{2}+1\right)}{\Gamma\left(k+\frac{m}{2}+s+1\right)} C .
$$

The functions $a\left(\xi^{2}\right)$ and $b\left(\xi^{2}\right)$ are then given by

$$
\begin{aligned}
a\left(\xi^{2}\right) & =C \sum_{s=0}^{\infty} \frac{(-1)^{s-1}}{2^{2 s-1} s !} \frac{\Gamma\left(k+\frac{m}{2}+1\right)}{\Gamma\left(k+\frac{m}{2}+s\right)} \xi^{2 s} \\
& =-2 C \Gamma\left(k+\frac{m}{2}+1\right) \sum_{s=0}^{\infty} \frac{(-1)^{s}}{s ! \Gamma\left(k+\frac{m}{2}+s\right)}\left(\frac{\xi}{2}\right)^{2 s} \\
& =-2 C \Gamma\left(k+\frac{m}{2}+1\right)\left(\frac{\xi}{2}\right)^{-\left(k+\frac{m}{2}-1\right)} J_{k+\frac{m}{2}-1}(\xi)
\end{aligned}
$$

and

$$
\begin{aligned}
b\left(\xi^{2}\right) & =C \Gamma\left(k+\frac{m}{2}+1\right) \sum_{s=0}^{\infty} \frac{(-1)^{s}}{s ! \Gamma\left(k+\frac{m}{2}+s+1\right)}\left(\frac{\xi}{2}\right)^{2 s} \\
& =C \Gamma\left(k+\frac{m}{2}+1\right)\left(\frac{\xi}{2}\right)^{-\left(k+\frac{m}{2}\right)} J_{k+\frac{m}{2}}(\xi)
\end{aligned}
$$


where

$$
J_{\alpha}(x)=\left(\frac{x}{2}\right)^{\alpha} \sum_{s=0}^{\infty} \frac{(-1)^{s}}{s ! \Gamma(s+\alpha+1)}\left(\frac{x}{2}\right)^{2 s}
$$

is the Bessel function of the first kind.

Now, choose $C$ such that $a(0)=1$, that is, $C=-\frac{1}{2} \frac{\Gamma\left(k+\frac{m}{2}\right)}{\Gamma\left(k+\frac{m}{2}+1\right)}$, then

$$
\begin{aligned}
& a\left(\xi^{2}\right)=2^{k+\frac{m}{2}-1} \Gamma\left(k+\frac{m}{2}\right) \xi^{1-k-\frac{m}{2}} J_{k+\frac{m}{2}-1}(\xi), \\
& b\left(\xi^{2}\right)=-2^{k+\frac{m}{2}-1} \Gamma\left(k+\frac{m}{2}\right) \xi^{-k-\frac{m}{2}} J_{k+\frac{m}{2}}(\xi) .
\end{aligned}
$$

We may conclude that

$$
2^{k+\frac{m}{2}-1} \Gamma\left(k+\frac{m}{2}\right)\left(e^{\xi_{0}} \xi^{1-k-\frac{m}{2}} J_{k+\frac{m}{2}-1}(\xi)-e^{-\xi_{0}} \xi^{1-k-\frac{m}{2}} J_{k+\frac{m}{2}}(\xi)\right) P_{k}(\xi)
$$

is an axially exponential monogenic function.

If $m$ is odd, then, we use that

$$
(2 n-1) ! !=2^{n} \Gamma\left(n+\frac{1}{2}\right) \sqrt{\pi}, \quad n \in \mathbb{N}
$$

so for $p$ odd: $p ! !=2^{\frac{p+1}{2}} \Gamma\left(\frac{p}{2}+1\right) \sqrt{\pi}$ :

$$
a_{s}=\frac{(-1)^{s-1}}{2^{s} s !} \frac{2^{k+\frac{m+1}{2}} \Gamma\left(k+\frac{m}{2}+1\right)}{2^{k+\frac{m}{2}+s-1+\frac{1}{2}} \Gamma\left(k+\frac{m}{2}+s-1+1\right)} C=\frac{(-1)^{s-1}}{2^{2 s-1} s !} \frac{\Gamma\left(k+\frac{m}{2}+1\right)}{\Gamma\left(k+\frac{m}{2}+s\right)} C,
$$

and

$$
b_{s}=\frac{(-1)^{s}}{2^{s} s !} \frac{2^{k+\frac{m+1}{2}} \Gamma\left(k+\frac{m}{2}+1\right)}{2^{k+\frac{m+1}{2}+s} \Gamma\left(k+\frac{m}{2}+s+1\right)} C=\frac{(-1)^{s}}{2^{2 s} s !} \frac{\Gamma\left(k+\frac{m}{2}+1\right)}{\Gamma\left(k+\frac{m}{2}+s+1\right)} C .
$$

We obtain the same coëfficiënts as in the case where $m$ is even. We obtain the same conclusion.

\section{Example 2}

If we look at axially monogenic functions where $A\left(0, \xi^{2}\right)=\exp \left(-\frac{\xi^{2}}{2}\right)$ and $B\left(0, \xi^{2}\right)=0$, we obtained a discrete monogenic function whose restriction to $\xi_{0}=0$ equals $\exp \left(-\frac{\xi^{2}}{2}\right) P_{k}$. We will thus obtain the Cauchy-Kovalevskaya extension, short CK-extension [11], of $\exp \left(-\frac{\xi^{2}}{2}\right) P_{k}$. We, however, already have established (for example [14]) that this CK-extension involves the Clifford-Hermite polynomials $H_{\ell, m, k}\left(P_{k}\right)$ :

$$
C K\left[\exp \left(-\frac{\xi^{2}}{2}\right) P_{k}\right]=\sum_{\ell=0}^{\infty} \frac{\xi_{0}^{\ell}}{\ell !} H_{\ell, m, k}(\xi) \exp \left(-\frac{\xi^{2}}{2}\right) P_{k} .
$$

We will now show that these Clifford-Hermite polynomials can also be obtained by means of the Vekua system. Set therefore

$$
\begin{aligned}
& A\left(\xi_{0}, \xi^{2}\right)=\sum_{\ell=0}^{\infty} \xi_{0}^{\ell} a_{\ell}\left(\xi^{2}\right) \exp \left(-\frac{\xi^{2}}{2}\right), \\
& B\left(\xi_{0}, \xi^{2}\right)=\sum_{\ell=0}^{\infty} \xi_{0}^{\ell} b_{\ell}\left(\xi^{2}\right) \exp \left(-\frac{\xi^{2}}{2}\right),
\end{aligned}
$$

where $a_{s}$ and $b_{s}$ are functions of $\xi^{2}$ with $a_{0}\left(\xi^{2}\right)=1$ and $b_{0}\left(\xi^{2}\right)=0$. We will show that from the Vekua system, we obtain that

$$
\left(A\left(\xi_{0}, \xi^{2}\right)+\xi B\left(\xi_{0}, \xi^{2}\right)\right) P_{k}=\mathrm{CK}\left[\exp \left(-\frac{\xi^{2}}{2}\right) P_{k}\right]=\sum_{\ell=0}^{\infty} \frac{\xi_{0}^{\ell}}{\ell !} H_{\ell, m, k}(\xi) \exp \left(-\frac{\xi^{2}}{2}\right) P_{k}
$$

or thus that

$$
\ell !\left(a_{\ell}+(-1)^{\ell} \xi b_{\ell}\right)=H_{\ell, m, k} .
$$

The Vekua system is given by 
If we take into consideration that

$$
\left\{\begin{array}{l}
\frac{d}{d \xi_{0}}(A)+\xi \frac{d}{d \xi}(B)+(2 k+m) B=0 \\
\frac{d}{d \xi}(A)-\xi \frac{d}{d \xi_{0}}(B)=0
\end{array}\right.
$$

$$
\begin{aligned}
\partial\left(b_{\ell}\left(\xi^{2}\right) \exp \left(-\frac{\xi^{2}}{2}\right)\right)[1] & =\sum_{s=0}^{\infty} \partial\left(\xi^{2 s} \exp \left(-\frac{\xi^{2}}{2}\right) b_{\ell}^{s}\right)[1] \\
& =\sum_{s=0}^{\infty}\left(2 s \xi^{2 s}+\xi^{2 s} \partial\right) \exp \left(-\frac{\xi^{2}}{2}\right)[1] b_{\ell}^{s} \\
& =\sum_{s=0}^{\infty} 2 s \xi^{2 s} \exp \left(-\frac{\xi^{2}}{2}\right)[1] b_{\ell}^{s}-\sum_{s=0}^{\infty} \xi^{2 s+1} \exp \left(-\frac{\xi^{2}}{2}\right)[1] b_{\ell}^{s} \\
& =\left(\partial\left(b_{\ell}\right)-\xi b_{\ell}\right) \exp \left(-\frac{\xi^{2}}{2}\right)[1],
\end{aligned}
$$

or thus

the first line translates to

$$
\frac{d}{d \xi}\left(b_{\ell}\left(\xi^{2}\right) \exp \left(-\frac{\xi^{2}}{2}\right)\right)=\left(\frac{d}{d \xi}\left(b_{\ell}\right)-\xi b_{\ell}\right) \exp \left(-\frac{\xi^{2}}{2}\right)
$$

$$
\left(\sum_{\ell=1}^{\infty} \ell \xi_{0}^{\ell-1} a_{\ell}+\sum_{\ell=0}^{\infty} \xi_{0}^{\ell}\left(\xi \frac{d}{d \xi}\left(b_{\ell}\right)-\xi^{2} b_{\ell}+(2 k+m) b_{\ell}\right)\right) \exp \left(-\frac{\xi^{2}}{2}\right)=0
$$

or equivalently

$$
(\ell+1) a_{\ell+1}+\xi \frac{d}{d \xi}\left(b_{\ell}\right)+\left(-\xi^{2}+(2 k+m)\right) b_{\ell}=0 .
$$

From this recurrence relation and $b_{0}=0$, we can immediately see that $a_{1}=0$.

The second line of the Vekua system can be equivalently expressed as

$$
\begin{aligned}
& \sum_{\ell=0}^{\infty}(-1)^{\ell} \xi_{0}^{\ell} \frac{d}{d \xi}\left(a_{\ell} \exp \left(-\frac{\xi^{2}}{2}\right)\right)-\sum_{\ell=1}^{\infty}(-1)^{\ell-1} \ell \xi_{0}^{\ell-1} \xi b_{\ell} \exp \left(-\frac{\xi^{2}}{2}\right)=0 \\
\Leftrightarrow & \frac{d}{d \xi}\left(a_{\ell}\right)-\xi a_{\ell}-(\ell+1) \xi b_{\ell+1}=0 .
\end{aligned}
$$

This immediately implies that $b_{2}=0$. Recursively, we will find that

$$
a_{2 \ell+1}=0, \quad b_{2 \ell}=0, \quad \forall \ell=0,1, \ldots
$$

We are thus left to show that

$$
\left\{\begin{array}{l}
a_{2 \ell}=\frac{1}{(2 \ell) !} H_{2 \ell, m, k} \\
\xi b_{2 \ell+1}=\frac{-1}{(2 \ell+1) !} H_{2 \ell+1, m, k} .
\end{array}\right.
$$

We prove this by induction on $\ell$. We will use (see [14]) that

$$
\left\{\begin{array}{l}
H_{2 \ell, m, k} P_{k}=(\partial-\xi) H_{2 \ell-1, m, k} P_{k} \\
H_{2 \ell+1, m, k} P_{k}=-(\partial-\xi) H_{2 \ell, m, k} P_{k}
\end{array}\right.
$$

We combine $a_{0}=1$ with (9) to find that $\xi b_{1}=-\xi=-\xi H_{1, m, k}$. From this and (8), it follows that $a_{2}=\frac{1}{2}\left(-\xi^{2}+(2 k+m)\right)=\frac{1}{2} H_{2, m, k}$. We now assume (induction hypothesis) that $a_{2 \ell-2}=\frac{1}{(2 \ell-2) !} H_{2 \ell-2, m, k}$. Then (9) shows

$$
\xi b_{2 \ell-1}=\frac{1}{(2 \ell-1)}\left(\frac{d}{d \xi}-\xi\right)\left(a_{2 \ell-2}\right) \text {. }
$$

Note that, because $H_{2 \ell-2, m, k}$ only includes even powers of $\xi$, we know that $\frac{d}{d \xi}\left(H_{2 \ell-2, m, k}\right) P_{k}=\partial\left(H_{2 \ell-2, m, k} P_{k}\right)$. We thus find that 


$$
\xi b_{2 \ell-1} P_{k}=\frac{1}{(2 \ell-1) !}\left(\frac{d}{d \xi}-\xi\right)\left(H_{2 \ell-2, m, k}\right) P_{k}=\frac{1}{(2 \ell-1) !}(\partial-\xi)\left(H_{2 \ell-2, m, k} P_{k}\right)=\frac{-1}{(2 \ell-1) !} H_{2 \ell-1, m, k} P_{k}
$$

from which it follows that $\xi b_{2 \ell-1}=\frac{-1}{(2 \ell-1) !} H_{2 \ell-1, m, k}$.

From (8), we see that

$$
a_{2 \ell} P_{k}=\frac{-1}{2 \ell}\left(\xi \frac{d}{d \xi}\left(b_{2 \ell-1}\right)+\left(-\xi^{2}+(2 k+m)\right) b_{2 \ell-1}\right) P_{k}
$$

Again, because $b_{2 \ell-1}\left(\xi^{2}\right)$ involves only even powers of $\xi$, we know that $\frac{d}{d \xi}\left(b_{2 \ell-1}\right) P_{k}=\partial\left(b_{2 \ell-1} P_{k}\right)$ and thus

$$
\begin{aligned}
a_{2 \ell} P_{k} & =\frac{-1}{2 \ell}\left(\xi \partial\left(b_{2 \ell-1} P_{k}\right)+\left(-\xi^{2}+(2 k+m)\right) b_{2 \ell-1} P_{k}\right)=\frac{-1}{2 \ell}\left((2 \Gamma-m+\partial \xi) b_{2 \ell-1} P_{k}+\left(-\xi^{2}+(2 k+m)\right) b_{2 \ell-1} P_{k}\right) \\
& =\frac{-1}{2 \ell}\left(2 b_{2 \ell-1} \Gamma P_{k}+\partial \xi b_{2 \ell-1} P_{k}+\left(-\xi^{2}+2 k\right) b_{2 \ell-1} P_{k}\right)=\frac{-1}{2 \ell}(\partial-\xi) \xi b_{2 \ell-1} P_{k}
\end{aligned}
$$

because $b_{2 \ell-1}$ is a function of $\xi^{2},\left[\Gamma, \xi^{2}\right]=0$ and $\Gamma P_{k}=-k P_{k}$. Because of the induction hypothesis, we may now substitute $\xi b_{2 \ell-1}=$ $\frac{-1}{(2 \ell-1) !} H_{2 \ell-1, m, k}$ and find

$$
(\partial-\xi) H_{2 \ell-1, m, k} P_{k}=H_{2 \ell, m, k} P_{k}
$$

so $a_{2 \ell} P_{k}=\frac{1}{(2 \ell) !} H_{2 \ell, m, k} P_{k}$ and $a_{2 \ell}=\frac{1}{(2 \ell) !} H_{2 \ell, m, k}$.

\section{Example 3}

Let again $\partial=\sum_{j=1}^{m} \partial_{j}$ and $\xi=\sum_{j=1}^{m} \xi_{j}$. The previous two examples are consistent with the corresponding results in the continuous setting. However, discrete Clifford-Hermite polynomials not only arise in the CK-extension of the function $\exp \left(-\frac{\xi^{2}}{2}\right) P_{k}(\xi)$ but also in the CK-extension of the discrete distribution $P_{k}(\xi) G$, where $G$ is the discrete Gaussian distribution $G=(2 \pi)^{\frac{m}{2}} \exp \left(\frac{\partial^{2}}{2}\right) \boldsymbol{\delta}_{0}$ and $P_{k}(\xi)$ is a discrete spherical monogenic operator of degree $k$, that is, a discrete homogeneous polynomial operator satisfying $\partial P_{k}[1]=0$, acting on the distribution $G$. Indeed, if we consider $G_{0}=G$ and determine discrete distributions $G_{s}$ such that

$$
\left(\partial_{0}+\partial\right)\left(\sum_{s=0}^{\infty} \frac{1}{s !} \xi_{0}^{s} P_{k}(\xi) G_{s}\right)=0
$$

then we find exactly the discrete Clifford-Hermite polynomials $H_{s, m, k}(\xi)$ :

$$
G_{s}=H_{s, m, k}(\xi) P_{k}(\xi) G
$$

The discrete distribution $G$, however, also has a discrete density function,that is, as a function $G$ can be viewed to equal the discrete function

$$
g\left(x_{1}, \ldots, x_{m}\right)=(2 \pi)^{\frac{m}{2}} \exp \left(\frac{\partial^{2}}{2}\right) \delta_{0}=\left(\frac{\sqrt{2 \pi}}{e}\right)^{m} \text { Bessell }\left(x_{1}, 1\right) \ldots \text { Bessell }\left(x_{m}, 1\right)
$$

where now $\delta_{0}$ is the discrete delta function and Bessell the modified Bessel function of the first kind. We may thus also consider the CK-extension of $g$. We will consider this for $m=1$ and for $P_{k}=1$. For the sake of simplicity, we will omit the factor $\frac{\sqrt{2 \pi}}{e}$.

The CK-extension of $g$ is given as

$$
C K[g]=\sum_{s=0}^{\infty} \frac{\xi_{0}^{s}}{s !} f_{s}
$$

where $f_{0}=g$ and $f_{s+1}=(-1)^{s+1} \partial_{1} f_{s}$. We, thus, obtain that

$$
\begin{aligned}
f_{2 \ell} & =(-1)^{\ell} \partial_{1}^{2 \ell} g=(-1)^{\ell} \sum_{t=0}^{\infty} \frac{\partial_{1}^{2 \ell+2 t}}{2^{t} t !} \delta_{0} \\
f_{2 \ell+1} & =(-1)^{\ell+1} \partial_{1}^{2 \ell+1} g=(-1)^{\ell+1} \sum_{t=0}^{\infty} \frac{\partial_{1}^{2 \ell+2 t+1}}{2^{t} t !} \delta_{0} .
\end{aligned}
$$

The one-dimensional Taylor series expansion of the discrete delta function is given by 


$$
\delta_{0}=\sum_{q=0}^{\infty} \frac{(-1)^{q}}{q ! q !} \xi_{1}^{2 q}[1]+\sum_{q=0}^{\infty} \frac{(-1)^{q+1}}{q !(q+1) !} \xi_{1}^{2 q+1}[1]\left(\mathbf{e}_{1}^{+}-\mathbf{e}_{1}^{-}\right)
$$

From this it follows that

$$
\begin{aligned}
\mathrm{CK}[g] & =\sum_{\ell, q^{\prime}=0}^{\infty} \frac{(-1)^{q^{\prime}}}{(2 \ell) !\left(2 q^{\prime}\right) !}\left[\sum_{t=0}^{\infty} \frac{(-1)^{t}}{2^{t} t !}\left(\begin{array}{c}
2 q^{\prime}+2 \ell+2 t \\
q^{\prime}+\ell+t
\end{array}\right)\right] \xi_{0}^{2 \ell} \xi_{1}^{2 q^{\prime}}[1] \\
& +\sum_{\ell, q^{\prime}=0}^{\infty} \frac{(-1)^{q^{\prime}+1}}{(2 \ell) !\left(2 q^{\prime}+1\right) !}\left[\sum_{t=0}^{\infty} \frac{(-1)^{t}}{2^{t} t !}\left(\begin{array}{c}
2 q^{\prime}+2 \ell+2 t+1 \\
q^{\prime}+\ell+t+1
\end{array}\right)\right] \xi_{0}^{2 \ell} \xi_{1}^{2 q^{\prime}+1}[1]\left(\mathbf{e}_{1}^{+}-\mathbf{e}_{1}^{-}\right) \\
& +\sum_{\ell, q^{\prime}=0}^{\infty} \frac{(-1)^{q^{\prime}}}{(2 \ell+1) !\left(2 q^{\prime}+1\right) !}\left[\sum_{t=0}^{\infty} \frac{(-1)^{t}}{2^{t} t !}\left(\begin{array}{c}
2 q^{\prime}+2 \ell+2 t+2 \\
q^{\prime}+\ell+t+1
\end{array}\right)\right] \xi_{0}^{2 \ell+1} \xi_{1}^{2 q^{\prime}+1}[1] \\
& +\sum_{\ell, q^{\prime}=0}^{\infty} \frac{(-1)^{q^{\prime}}}{(2 \ell+1) !\left(2 q^{\prime}\right) !}\left[\sum_{t=0}^{\infty} \frac{(-1)^{t}}{2^{t} t !}\left(\begin{array}{c}
2 q^{\prime}+2 \ell+2 t+1 \\
q^{\prime}+\ell+t+1
\end{array}\right)\right] \xi_{0}^{2 \ell+1} \xi_{1}^{2 q^{\prime}}[1]\left(\mathbf{e}_{1}^{+}-\mathbf{e}_{1}^{-}\right) \\
= & A\left(\xi_{0}, \xi^{2}\right)+\xi_{1} B\left(\xi_{0}, \xi_{1}^{2}\right)
\end{aligned}
$$

where

$$
\begin{aligned}
A\left(\xi_{0}, \xi_{1}^{2}\right)= & \sum_{\ell, q=0}^{\infty} \frac{(-1)^{q}}{(2 \ell) !(2 q) !}\left[\sum_{t=0}^{\infty} \frac{(-1)^{t}}{2^{t} t !}\left(\begin{array}{c}
2 q+2 \ell+2 t \\
q+\ell+t
\end{array}\right)\right] \xi_{0}^{2 \ell} \xi_{1}^{2 q}[1] \\
& +\sum_{\ell, q=0}^{\infty} \frac{(-1)^{q}}{(2 \ell+1) !(2 q) !}\left[\sum_{t=0}^{\infty} \frac{(-1)^{t}}{2^{t} t !}\left(\begin{array}{c}
2 q+2 \ell+2 t+1 \\
q+\ell+t+1
\end{array}\right)\right] \xi_{0}^{2 \ell+1} \xi_{1}^{2 q}[1]\left(\mathbf{e}_{1}^{+}-\mathbf{e}_{1}^{-}\right) \\
= & \sum_{\ell, q=0}^{\infty} \frac{(-1)^{\ell}}{(2 \ell) !(2 q) !} \xi_{0}^{2 \ell} \xi_{1}^{2 q}[1]\left(\partial_{1}^{2 q+2 \ell} g\right)(0) \\
& +\sum_{\ell, q=0}^{\infty} \frac{(-1)^{\ell+1}}{(2 \ell+1) !(2 q) !} \xi_{0}^{2 \ell+1} \xi_{1}^{2 q}[1]\left(\partial_{1}^{2 q+2 \ell+1} g\right)(0) \\
B\left(\xi_{0}, \xi_{1}^{2}\right)= & \sum_{\ell, q=0}^{\infty} \frac{(-1)^{q+1}}{(2 \ell) !(2 q+1) !}\left[\sum_{t=0}^{\infty} \frac{(-1)^{t}}{2^{t} t !}\left(\begin{array}{c}
2 q+2 \ell+2 t+1 \\
q+\ell+t+1
\end{array}\right)\right] \xi_{0}^{2 \ell} \xi_{1}^{2 q}[1]\left(\mathbf{e}_{1}^{+}-\mathbf{e}_{1}^{-}\right) \\
& +\sum_{\ell, q=0}^{\infty} \frac{(-1)^{q+1}}{(2 \ell+1) !(2 q+1) !}\left[\sum_{t=0}^{\infty} \frac{(-1)^{t}}{2^{t} t !}\left(\begin{array}{c}
2 q+2 \ell+2 t+2 \\
q+\ell+t+1
\end{array}\right)\right] \xi_{0}^{2 \ell+1} \xi_{1}^{2 q}[1] \\
& =\sum_{\ell, q=0}^{\infty} \frac{(-1)^{\ell}}{(2 \ell) !(2 q+1) !} \xi_{0}^{2 \ell} \xi_{1}^{2 q}[1]\left(\partial_{1}^{2 q+2 \ell+1} g\right)(0) \\
& +\sum_{\ell, q=0}^{\infty} \frac{(-1)^{\ell}}{(2 \ell+1) !(2 q+1) !} \xi_{0}^{2 \ell+1} \xi_{1}^{2 q}[1]\left(\partial_{1}^{2 q+2 \ell+2} g\right)(0)
\end{aligned}
$$

because

$$
\begin{aligned}
f_{2 q+2 \ell}(0) & =\sum_{t=0}^{\infty} \frac{(-1)^{t}}{2^{t} t !}\left(\begin{array}{c}
2 \ell+2 q+2 t \\
q+\ell+t
\end{array}\right)=(-1)^{q+\ell}\left(\partial_{1}^{2 q+2 \ell} g\right)(0) \\
f_{2 q+2 \ell+1}(0) & =\sum_{t=0}^{\infty} \frac{(-1)^{t}}{2^{t} t !}\left(\begin{array}{c}
2 q+2 \ell+2 t+1 \\
q+\ell+t+1
\end{array}\right)\left(\mathbf{e}_{1}^{+}-\mathbf{e}_{1}^{-}\right)=(-1)^{q+\ell+1}\left(\partial_{1}^{2 q+2 \ell+1} g\right)(0) .
\end{aligned}
$$

One can easily check that $A$ and $B$ satisfy the Vekua-system:

$$
\left\{\begin{array}{l}
\partial_{0}(A)+\xi \partial(B)+m B=0 \\
\partial(A)-\xi \partial_{0}(B)=0
\end{array}\right.
$$

\section{Conclusion and future research}

In this paper, we found that two classical techniques for the construction of axially monogenic polynomials in Euclidean Clifford analysis may be readily discretized to the Hermitian discrete Clifford analysis setting. A first discretization is a discrete Fueter's theorem, allowing 
the extension of a two-dimensional discrete monogenic function to $m$ dimenisons ( $m$ odd) by the action of a certain power of the star Laplacian on the said function and (if necessary) a discrete monogenic $P_{k}$. A second technique starts from the definition of discrete axially monogenic functions

$$
\left(A\left(\xi_{0}, \xi^{2}\right)+\xi B\left(\xi_{0}, \xi^{2}\right)\right) P_{k}
$$

and derives a Vekua-type system, which the functions $A\left(\xi_{0}\right)$ and $B\left(\xi_{0}\right)$, considered radial in $\xi$ and having real Taylor coefficients, have to satisfy.

A topic for future research and a nice application would involve a factorization of the discrete Heat equation (see [12]) by means of a parabolic Dirac operator, which may be considered as an extension of the Dirac operator with an additional time dimension, and applying (a slightly modified version of) Fueters theorem to obtain solutions to the first-order Clifford-Heat equation. Moreover, the use of axially monogenic special functions such as the Clifford-Hermite polynomials may be a starting point for the study of wavelet transforms in the discrete setting, as has been performed in the continuous setting in [15].

\section{Acknowledgement}

The first author acknowledges the support of the Research Foundation - Flanders (FWO) (grant FWO13/PDO/039).

\section{References}

1. Fueter R. Die funktionentheorie der Differentialgleichungen $\Delta u=0$ und $\Delta \Delta u=0$ mit vier reellen variablen. Commentarii mathematici Helvetici 1935; 7:307-330.

2. Sce M. Osservazioni sulle serie di potenze nei moduli quadratici. Atti della Accademia Nazionale dei Lincei. Classe di Scienze Fisiche, Matematiche e Naturali 1957; 8(23):220-225.

3. Qian T. Generalization of Fueter's result to $\mathbb{R}^{n+1}$. Atti della Accademia Nazionale dei Lincei. Classe di Scienze Fisiche, Matematiche e Naturali. Rendiconti Lincei. Matematica e Applicazioni 1997; 8(2):111-117.

4. Kou KI, Qian T, Sommen F. Generalizations of Fueter's theorem. Methods and Applications of Analysis 2002; 9(2):273-289.

5. Sommen F. On a generalization of Fueter's theorem. Zeitschrift für Analysis und ihre Anwendungen 2000; 19(4):899-902.

6. Brackx F, Delanghe R, Sommen F. Clifford Analysis, Research Notes in Mathematics, vol. 76. Pitman Publishers: Boston-London-Melbourne, 1982.

7. Delanghe R, Sommen F, Souček V. Clifford Algebras and Spinor-Valued Functions. Kluwer Academic Publishers: Dordrecht, The Netherlands, 1992.

8. Delanghe R. Clifford analysis: history and perspective. Computational Methods and Function Theory 2001; 1(1):107-153.

9. De Schepper H, Sommen F, Van de Voorde L. A basic framework for discrete Clifford analysis. Experimental Mathematics 2009; 18(4):385-395.

10. De Ridder H, De Schepper H, Sommen F, Kähler U. Discrete function theory based on skew Weyl relations. Proceedings of the American Mathematical Society 2010; 138(9):3241-3256.

11. De Ridder H, De Schepper H, Sommen F. The Cauchy-Kovalevskaya extension theorem in discrete Clifford analysis. Communications on Pure and Applied Analysis 2011; 10(4):1097-1109.

12. Baaske F, Bernstein S, De Ridder H, Sommen F. On solutions of a discretized heat equation in discrete Clifford analysis. Journal of Differential Equations and Applications 2014; 20(2):271-295.

13. De Ridder H, De Schepper H, Sommen F. Fueter polynomials in discrete Clifford analysis. Mathematische Zeitschrift 2012; 272(1-2):253-268.

14. De Ridder H. Discrete Clifford Analysis: Function Theory and Integral Transforms. Ghent University: Ghent, Belgium, 2013.

15. Brackx F, De Schepper H, De Schepper N, Sommen F. Generalized Hermitean Clifford-Hermite polynomials and the associated wavelet transform. Mathematical Methods in the Applied Sciences 2009; 32(5):606-630. 